\title{
PROPERTIES OF COMPLEMENTS IN THE LATTICE OF CONVERGENCE STRUCTURES
}

\author{
C.V. RIECKE \\ Department of Mathematics \\ Cameron University \\ Lawton, Oklahoma 73505 U.S.A. \\ (Received October 11, 1979)
}

ABSTRACT. Relative complements and differences are investigated for several convergence structure lattices, especially the lattices of Kent convergence structures and the lattice of pretopologies. Convergence space properties preserved by relative complementation are studied. Mappings of some convergence structure lattices into related lattices of lattice homomorphisms are considered.

KEY WORDS AND PHRASES. Convergence structure, pretopology, limitierung, relative pseudo-complement, pseudo-difference, continuous lattice.

1980 MATHEMATICS SUBJECT CLASSIFICATION CODES. 54A05, 06015.

1. INTRODUCTION.

The author classified the relative complements and differences for the lattice of Kent convergence structures on a nonempty set in [8]. This paper investigates further the convergence space properties preserved by these complements and their relationships to some of the standard lattice operations such as products 
and quotients.

The definitions used are essentially those of [3], [4] and [11] with a convergence structure on $X$ considered as a map $q: X \rightarrow \beta(F(X)$ ) (the power set of the set of filters on $X) . \quad U(X)$ and $\dot{x}$ are the set of ultrafilters on $X$ and the principal ultrafilter generated by $\{x\}$. For a convergence space $(x, q)$, let $\lambda q, \phi q$ and $\omega q$ be the topological, pretopological, and completely regular topological modifications of $q$. The $q$-limit set of a filter $F$ is ad $q(F)=\{x \mid F \in q(x)\}$ and the closure $c 1(A)$ of a subset $A$ is $\{x \mid F \in q(x)$ for some filter $F$ with $A \in F\}$.

An element $z$ of lattice $L$ is the pseudo-complement of $x$ relative to $y$ ( $x * y)$ if $z$ is the greatest element with $x \wedge z \leq y$ and the pseudo-difference of $y$ and $x(y-x)$ if $z$ is the least element with $y \leq x \vee z$. If $L$ is a complete lattice with 0 and 1 the least and greatest elements, then the pseudo-complement of $x$ is $x^{*}=x * 0$ and the pseudo-difference of $x$ is $-x=1-x$ (a change of notation from [8]).

\section{RELATIVE COMPLEMENTS.}

The relative pseudo-complement and pseudo-difference of two convergence structures in $\mathrm{C}(\mathrm{X})$ were described in [8] as:

$$
\begin{aligned}
& q \star r(x)=\{F \mid F=\dot{x} \text { or } G \cap \dot{x} \subseteq F \text { for some } G \in r(x) \backslash q(x)\} \\
& q-r(x)=\{F \mid F+G=\beta(x) \text { or is in } q(x) \text { for all } G \in r(x)\}
\end{aligned}
$$

For $\mathrm{q}$ and $\mathrm{r}$ limitierungs of Fischer [3] or pseudo-topologies the same descriptions hold for relative pseudo-complements and pseudo-differences in the lattices of limitierungs or pseudotopologies. In $\mathrm{P}(\mathrm{X})$, the lattices of pretopologies on X, pseudo-differences do not exist from [9]. Relative pseudo-complements also fail to exist in $P(X)$ even though, from [9], $P(X)$ is pseudo-complemented.

EXAMPLE 2.1: On an infinite set $X$, let $A$ be an infinite subset with infinite complement and $x \in X$. If $q(r)$ is the finest pretopology on $X$ such that an ultrafilter $F q(r)$-converges to $x$ if and only if $F=\dot{x}$ or $F$ is free and contains $A(X-A)$, then $q * r$ does not exist in $P(X)$. 
Many properties of convergence structures are preserved by relative pseudocomplementation. If $\mathbf{r}$ is a limitierung (resp. pseudotopology, pretopology, topology), then from [8], $q * r$ is the same type of structure for any convergence structure q.

PROPOSITION 2.2: For any convergence structures $q$ and $r$ on $x$ :

(i) $q * r$ is $T_{1}$ (Hausdorff) if $r$ is $T_{1}$ (Hausdorff).

(ii) $q * r$ is $T_{3}$ if $r$ is $T_{3}$.

(iii) $q * r$ is compact if and only if $r$ is compact and for any ultrafilter $F, \operatorname{ad}_{\mathbf{r}}(G) \notin \operatorname{ad}_{q}(G)$ for some $G \subset F$.

(iv) $q * r$ is $T$-regular [5] if $r$ is $T$-regular.

(v) $q * r$ is first countable $(\boldsymbol{N}$-countable, [2]) if $r$ is first countable $(Y$-countable).

(vi) $q * r$ is second countable if $r$ is second countable and $q * r$ has at most countably many discrete points.

In addition, for any pretopology $q$ on $x$ :

(vii) $q * r$ is a completely regular topology if $r$ is a completely regular topology.

(viii) $q * r$ is $\omega$-regular [6] if $r$ is $\omega$-regular.

(ix) $q * r$ is C-embedded [6] if $r$ is C-embedded.

PROOF: The proof of (i) is in [8].

(ii) If $F \in q * r(x)$ with $G \subseteq F$ for some $G \in \mathbf{r}(x) \backslash q(x)$ then $c l_{r} G \in r(x) \backslash q(x)$ since $r$ is regular so $\mathrm{cl}_{\mathrm{r}} G \subseteq \mathrm{cl}_{\mathrm{q} * \mathrm{r}} G \subseteq \mathrm{cl}_{\mathrm{q} * \mathrm{r}} F$ and $\mathrm{cl}{ }_{\mathrm{q} * \mathrm{r}} F \mathrm{q* \textrm {r } - \text { converges }}$ to $x$. If $q * r(x)=\{\dot{x}\}$ then $q * r$ is $T_{1}$ so $c l_{q * r} \dot{x}=\dot{x}$.

(iii) is obvious.

(iv) Suppose $F \in q * r(x)$ and $G \subseteq F$ for some $G \in r(x) \backslash q(x)$. Then $c 1, r^{G} \epsilon$ $r(x) \backslash q(x)$ so $c l_{\lambda r} G \subseteq c l_{q * \lambda r}{ }^{G} \subseteq c l_{\lambda(q * r)}{ }^{G} \subseteq c l_{\lambda(q * r)} F$ since $q * \lambda r \leq \lambda(q * r)$. If $q * r(x)=\{\dot{x}\}, \lambda(q * r)(x)=\{\dot{x}\}$ so $q * r$ is $T$-regular. 
(v) If $F \in q * r(x)$ with $G \subseteq F$ and $G \in r(x) \backslash q(x)$, then $H \in r(x) \backslash q(x)$ for some $H$ with filterbase of cardinality less than $N$ for any cardinal $N$.

(vi) Let $B$ be a countable basis for $(x, r)$. Then $B^{\prime}=B \cup\{x \mid q \star r(x)=\{\dot{x}\}\}$ is a countable basis for $(X, q * r)$.

(vii) Since $q * r$ is topological from [8], suppose $A$ is $q * r-c l o s e d$ and $x \notin A$. Then if $x \notin c 1_{r}(A)$, any real valued continuous function on $(x, r)$ which separates $x$ and $A$ is also $q \star r$-continuous. If $x \in c 1_{r}(A)$, then $q \star r$ is discrete at $x$ so $x$ and $A$ can be separated by a $q \star r-c o n t i n u o u s$, realvalued function.

(viii) If $F \in q * r(x)$ and $G \in r(x) \backslash q(x)$ with $c 1{ }_{\omega r} G \in r(x) \backslash q(x)$ then from (vii), if $\omega r$ is the completely regular modification of $r, c 1{ }_{\omega r} G \subseteq$ $c 1_{q * \omega r} G \subseteq c l_{\omega(q * r)^{G} \subseteq c 1_{\omega(q * r)}} F$ and if $q * r$ is discrete at $x$, so is $\omega(q * r)$ and the conclusion follows.

(ix) From [8], $q * r$ is pseudo-topological if $r$ is pseudo-topological. If $r$ is Hausdorff and $w$-regular, then $q * r$ has the same properties from (i) and (viii) so by [6], $q * r$ is C-embedded if $r$ is C-embedded.

COROLLARY 2.3: (i) If $\mathrm{r}^{0}$ is the finest first countable structure coarser than $r$, then $(q * r)^{0}=q * r^{0}$ for every convergence structure $q$.

(ii) If $\mathrm{Rr}$, the finest regular structure coarser than $r[7]$, is $T_{1}$, then $q * R r \leq R(q * r)$.

PROOF: Since $\mathrm{r}^{\circ} \leq \mathrm{r}, \mathrm{q} * \mathrm{r}^{\circ} \leq \mathrm{q} * \mathrm{r}$ and $\mathrm{q} * \mathrm{r}^{\circ}$ being first countable implies $\mathrm{q} * \mathrm{r}^{\circ}$ $\leq(q * r)^{0}$. Conversely, if $F \in q * r^{0}(x)$ then $G \subseteq F$ for some $G \in r(x) \backslash q(x)$ with countable filterbase. Then $G \in q * r(x)$ so $F \in(q * r)^{\circ}(x)$.

As $q * R r$ is $T_{3}$ from (ii) of Proposition 2.2 and $q * R r \leq q * r$, then $q * R r \leq R(q * r)$. The converses of the statements in Proposition 2.2 fail to be true since if $q \leq r, q \star r$ is discrete. In (ii) of Proposition 2.2, one cannot substitute regular for $\mathrm{T}_{3}$. 
EXAMPLE 2.4: (i) Let $X=\{x, y\}$ and $q$ be the finest convergence structure on $\mathrm{X}$ for which the principal filter $F_{\mathrm{xy}}$ generated by $\{\mathrm{x}, \mathrm{y}\}$ converges to $x$. Then 0 is regular but not $T_{1}$ and $q^{*}$ is not regular.

(ii) Let $r$ be a convergence structure on an infinite set $X$ for which $\operatorname{Rr} \neq r$ and $R r$ is $T_{1}$, such as a non-regular $T_{2}$-convergence structure which is finer than some $T_{2}$, regular topology. Then $1=R(r * r) \neq r * R r$.

The following description of the convergent ultrafilters of the pseudo-difference $q-r$ of two convergence structures is given in [8]:

LEMMA 2.5: An ultrafilter $F$ q-r converges to $x$ if and only if $F$-converges to $\mathbf{x}$ or does not $r$-converge to $\mathbf{x}$.

Because $q-r$ can have so many convergent ultrafilters, most convergence space properties are not preserved. This can also be observed from the result of [9] that the image of the map $q \rightarrow 1-q$ is the lattice of pseudotopologies. For example, one can readily show that $q-r$ is not pretopological if $q \notin r, r$ is $T_{1}$ and $q$ is not discrete and $1-q$ is not regular if $q$ is $T_{1}$ and not discrete. A few properties can be easily seen to be preserved.

PROPOSITION 2.6: For any convergence structures $q$ and $r$ on $x$,

(i) $q-r$ is $T_{1}$ if and only if $q$ is $T_{1}$ and the pretopological modification $\phi r$ of $r$ is indiscrete.

(ii) $q-r$ is Hausdorff if and only if $1-q \leq r$.

(iii) $q-r$ is compact if $q$ is compact.

(iv) $q-r$ is compact if and only if no ultrafilter $F(1-r) \wedge q$-converges to every point.

For complements of product convergence structures there exist relationships to the complements in the original spaces. If $\left\{\left(x_{\alpha}, q_{\alpha}\right) \mid \alpha \in \Gamma\right\}$ is a family of nondegenerate convergence spaces with products $\left(\pi x_{\alpha}, \pi q_{\alpha}\right)$, let $\pi_{w} q_{\alpha}$ denote the convergence structure defined on $\pi x_{\alpha}$ by: $F \Pi_{w} q_{\alpha}$-converges to $x=\left(x_{\alpha}\right)$ if and 
only if the projection $p_{\gamma}(F) q_{\gamma}$-converges to $x_{\gamma}$ for some $\gamma \in \Gamma$. $\Pi_{w} q_{\alpha}$ will be called the weak product convergence structure. In the subsequent four propositions, $\mathrm{p}$ or $\mathrm{p}_{\alpha}$ will denote the appropriate projection or quotient map.

PROPOSITION 2.7: If $q_{\alpha}$ and $r_{\alpha}$ are convergence structures on $x_{\alpha}$ for $\alpha \in \Gamma$ with $|\Gamma|>1$, then in $\mathrm{C}\left(\pi \mathrm{x}_{\alpha}\right)$ :

(i) $\left(\pi q_{\alpha}\right) *\left(\pi r_{\alpha}\right) \leq \Pi\left(q_{\alpha} * r_{\alpha}\right)$.

(ii) F converges to $x=\left(x_{\alpha}\right)$ with respect to $\left(\Pi q_{\alpha}\right) *\left(\pi r_{\alpha}\right)$ if and only if $p_{\gamma}(F) q_{\gamma} * r=$-converges to $x_{\gamma}$ for some $\gamma \in \Gamma$.

(iii) $\left(\Pi_{\alpha}\right) *\left(\pi r_{\alpha}\right)=\Pi_{w}\left(q_{\alpha} * r_{\alpha}\right)$.

(iv) $\Pi\left(\mathrm{q}_{\alpha} * \mathrm{r}_{\alpha}\right)=\left(\Pi \mathrm{q}_{\alpha}\right) *\left(\pi \mathrm{r}_{\alpha}\right)$ if and only if each $\mathrm{r}_{\alpha}$ is indiscrete.

(v) $\left(\Pi_{\alpha}\right)^{*}=\Pi_{w} q_{\alpha}^{*}$.

PROOF: (i) If $F \Pi\left(q_{\alpha} \star r_{\alpha}\right)$-converges to $x=\left(x_{\alpha}\right)$ then each $p_{\alpha}(F) q_{\alpha} \star r{ }_{\alpha}$-converges to $\mathrm{x}_{\alpha}$ so for each $\alpha$ there exists a filter $G_{\alpha}$ on $\mathrm{x}_{\alpha}$ with $G_{\alpha}=$ $\mathrm{p}_{\alpha}(F)=\dot{\mathrm{x}}_{\alpha}$ or $G_{\alpha} \subseteq \mathrm{p}_{\alpha}(F)$ and $G_{\alpha} \in \mathrm{r}_{\alpha}\left(\mathrm{x}_{\alpha}\right) \backslash \mathrm{q}_{\alpha}\left(\mathrm{x}_{\alpha}\right)$. Then $\pi G_{\alpha}$ is $\pi \mathrm{r}_{\alpha}$-convergent to $x$ and $\Pi G_{\alpha} \in\left(\pi r_{\alpha}\right)(x) \backslash\left(\pi q_{\alpha}\right)(x)$ or $\Pi G_{\alpha}=\dot{x}$ and $F\left(\pi q_{\alpha}\right) *\left(\Pi r_{\alpha}\right)-$ converges to $\mathrm{x}$ since $\pi G_{\alpha} \subseteq \Pi_{\alpha}(F) \subseteq F$.

(ii) Suppose $F\left(\pi q_{\alpha}\right) *\left(\pi r_{\alpha}\right)$-converges to $x=\left(x_{\alpha}\right)$. Then $F=\dot{x}$ or $G \subseteq F$ for some $G \in\left(\Pi r_{\alpha}\right)(x) \backslash\left(\pi q_{\alpha}\right)(x)$. In the latter case, $p_{\gamma}(G) \in r_{\gamma}\left(x_{\gamma}\right) \backslash q_{\gamma}\left(x_{\gamma}\right)$ for some $\gamma$ so $p_{\gamma}(F) \in q_{\gamma} * r_{\gamma}\left(x_{\gamma}\right)$. The converse is similar.

(iii) follows immediately from (ii) and the definition of a weak product.

(iv) If $|\Gamma|>1$ and $\pi q_{\alpha} \leq \pi_{w} q_{\alpha}$ then for $F_{\gamma}$ any filter on $x_{\gamma}$ and $x_{\gamma} \in x_{\gamma}$, let $G=\Pi G_{\alpha}$ where $G_{\alpha}=\dot{x}_{\alpha}$ for some $x_{\alpha} \in x_{\alpha}$ if $\alpha \neq \gamma$ and $G_{\gamma}=F_{\gamma}$. Then $G$ $\left(\pi_{w} q_{\alpha}\right)$-converges to $x=\left(x_{\alpha}\right)$ so must $\pi q_{\alpha}$-converge to $x$ and $F_{\gamma}=p_{\gamma}(G)$ $\mathrm{q}_{\gamma}$-converges to $\mathrm{x}_{\gamma}$ and $\mathrm{q}_{\gamma}$ is indiscrete. Thus if $\pi_{\alpha} * \mathrm{r}_{\alpha} \leq \pi_{w} \mathrm{q}_{\alpha} * \mathrm{r}_{\alpha}$, each $q_{\alpha} * r_{\alpha}$ is indiscrete and it follows that each $r_{\alpha}$ is indiscrete (since $\left\{\mathrm{x}_{\alpha}\right\} \in \mathrm{r}_{\alpha}\left(\mathrm{x}_{\alpha}\right) \backslash \mathrm{q}_{\alpha}\left(\mathrm{x}_{\alpha}\right)$ for each $\mathrm{x}_{\alpha} \in \mathrm{x}_{\alpha}$ ).

(v) is a direct consequence of (iv) since $q^{*}=q * 0$. 
PROPOSITION 2.8: If $\mathrm{q}_{\alpha}$ and $\mathrm{r}_{\alpha}$ are convergence structures on $\mathrm{x}_{\alpha}$ for $\alpha \in \Gamma$, then in $\mathrm{C}\left(\operatorname{\Pi x}_{\alpha}\right)$ :

(i) $\left(\pi q_{\alpha}\right)-\left(\pi r_{\alpha}\right) \leq \pi\left(q_{\alpha}-r_{\alpha}\right)$

(ii) $-\left(\Pi r_{\alpha}\right)$ and $\Pi_{w}\left(-r_{\alpha}\right)$ agree on convergent ultrafilters.

PROOF: (i) If $x=\left(x_{\alpha}\right)$ and $p_{\alpha}(F) q_{\alpha}-r_{\alpha}$ converges to $x_{\alpha}$ for all $\alpha$, let $G F$ $\left(\pi r_{\alpha}\right)(x)$. Then $\mathrm{p}_{\alpha}(G) \in \mathrm{r}_{c !}\left(\mathrm{x}_{\alpha}\right)$ for all $\alpha$, so $\mathrm{p}_{\alpha}(F)+\mathrm{p}_{\alpha}(G) \in \mathrm{q}_{\alpha}\left(\mathrm{x}_{\alpha}\right)$ or $\mathrm{p}_{\alpha}(F)+\mathrm{p}_{\alpha}(G)=\beta\left(\mathrm{X}_{\alpha}\right)$. In either case, $\mathrm{p}_{\alpha}(F)+\mathrm{p}_{\alpha}(G) \subseteq \mathrm{p}_{\alpha}(F+G)$ and $F+G \epsilon$ $\left[\left(\Pi_{\alpha}\right)-\left(\Pi r_{\alpha}\right)\right](x)$.

(ii) Let $F$ be an ultrafilter in $\left[-\left(\Pi r_{\alpha}\right)\right](\mathrm{x})$. Then $\mathrm{p}_{\alpha}(F) \notin \mathrm{r}_{\alpha}\left(\mathrm{x}_{\alpha}\right)$ for some $\alpha$ so $\mathrm{p}_{\alpha}(F) \in\left(-\mathrm{r}_{\alpha}\right)\left(\mathrm{x}_{\alpha}\right)$ and $F \Pi_{\mathrm{w}}\left(-\mathrm{r}_{\alpha}\right)$-converges to $\mathrm{x}$. The reverse inequality is similar.

From Lemma 2.5, one can show that equality does not hold in Proposition 2.8(i) even for q topological.

The product operation can also be viewed as a lattice operation on $\mathrm{C}(\mathrm{X})$.

PROPOSITION 2.9: The map $\Pi: \Pi\left[\mathrm{C}\left(\mathrm{X}_{\alpha}\right)\right] \rightarrow \mathrm{C}\left(\pi \mathrm{X}_{\alpha}\right)$ defined by $\Pi\left[\left(\mathrm{X}_{\alpha}, \mathrm{q}_{\alpha}\right)(\alpha \in \Gamma)\right]=$ $\left(\Pi \mathrm{X}_{\alpha}, \Pi \mathrm{q}_{\alpha}\right)$ is a complete join homomorphism.

If $(X, q)$ is a convergence space with $\sim$ an equivalence relation on $X, 1 e t x / \sim$ be the quotient space with quotient structure $\bar{q}, \bar{A}=\{\bar{x} \mid x \in A\}$ for $A c X$ and, for $F$ a filter on $X, \bar{F}=\{\bar{A} \mid A \in F\}$. Let $f: C(X) \rightarrow C(X ; \sim)$ be the map $f(q)=\bar{q}$. The subsequent propositions are readily established.

PROPOSITION 2.10: $\mathrm{f}$ is a complete meet homomorphism.

PROPOSITION 2.11: For $\mathrm{q}$ and $\mathrm{r}$ in $\mathrm{C}(\mathrm{X})$, in $\mathrm{C}(\mathrm{X} / \sim)$ :

(i) $\overline{\mathrm{q} * \mathrm{r}} \leq \overline{\mathrm{q}} * \overline{\mathrm{r}}$

(ii) $\overline{r^{*}}=\overline{(r)} *$ if and only if for each $x$ and $F$ in $r(x)$, there does not exist $A \in F$ with $A \cap \bar{y} \neq \phi$ for all $y$ in $X$.

(iii) $-\bar{q} \leq(\overline{-q})$ with equaljty if and only if for each $x, F \neq \dot{x}$ in $q(x)$ and $A \in F, A \notin \bar{x}$. 
(iv) If $q$ and $r$ are pretopologies, $\bar{q} \star \bar{r}=\overline{q * r}$ if $y \sim x$ and $\overline{N_{r}(y)}=\overline{N_{q}(x)}$ implies $N_{r}(y)=N_{q}(x)$.

For $A$ a nonempty subset of $X$ and $F$ a filter on $X$ with $A \in F$, let $F_{A}$ be the filter on $A$ where $F_{A}=\{A \cap B \mid B \in F\}$ and $f_{A}: C(X) \rightarrow C(A)$ be $f_{A}(q)(x)=\left\{F_{A} \mid\right.$ $A \in F$ and $F \in q(x)\}$, i.e., $f_{A}(q)$ is the subspace structure on $A$.

PROPOSITION 2.12: (i) $\mathrm{f}_{\mathrm{A}}$ is a complete lattice epimorphism.

(ii) For any $q$ and $r$ in $C(X), f_{A}(q * r)=f_{A}(q) * f_{A}(r)$ and $f_{A}(q-r)=f_{A}(q)-f_{A}(r)$. As one would expect, Proposition 2.12 establishes that the restriction of the relative complements to a subspace are the complements of the restrictions. 3. LATTICE OPERATORS INDUCED BY RELATIVE COMPLEMENTS.

The relative pseudo-complement and pseudo-difference induce four obvious self-maps of $\mathrm{C}(\mathrm{X})$ for each convergence structure $\mathrm{q}$ :

(i) $\mathrm{f}^{*}(\mathrm{q}): \mathrm{f}^{*}(\mathrm{q})(\mathrm{r})=\mathrm{q} * \mathrm{r}$

(ii) $\mathrm{f}_{*}(\mathrm{q}): \mathrm{f}_{*}(\mathrm{q})(\mathrm{r})=\mathrm{r} * \mathrm{q}$

(iii) $\mathrm{f}^{-}(\mathrm{q}): \mathrm{f}^{-}(\mathrm{q})(\mathrm{r})=\mathrm{q}-\mathrm{r}$

(iv) $f_{-}(q): f_{-}(q)(r)=r-q$

Of these maps, (i) and (iv) were considered in [8]. Only (i) and (iv) will be considered here since (ii) and (iii) have similar lattice properties if considered as maps of $\mathrm{C}(\mathrm{X})$ into its dual.

If $\Gamma$ is a cardinal, a subset $A$ of a lattice $L$ is prime with respect to $\Gamma$ joins in $L$ if for any subset $\left\{x_{\gamma} \mid \gamma \in \Gamma\right\}$ with $v_{x_{\gamma}} \in A$, some $x_{\gamma} \in A$. A convergence structure $q$ of $C(X)$ is join prime if each $q(x) \backslash\{\dot{x}\}$ is prime with respect to finite joins in $\{r(x) \backslash\{\dot{x}\} \mid r \in C(X)\}$. As an extension of a result in [8] one has:

PROPOSITION 3.1: For any convergence structure $q$ on $\mathrm{X}$ :

(i) $\mathrm{f}^{*}(\mathrm{q})$ is a complete meet homomorphism.

(ii) $\mathrm{f}^{*}(\mathrm{q})$ is a $\Gamma$-join homomorphism if and only if $\mathrm{q}(\mathrm{x}) \backslash\{\dot{\mathrm{x}}\}$ is prime with 


\section{respect to $\Gamma$-joins in $F(X)$ for any cardinal $\Gamma$. \\ (iii) $\mathrm{f}^{*}(\mathrm{q})$ is bijective if and only if $\mathrm{q}$ is discrete.}

PROOF: (i) is a result of [8] while the proof of (ii) parallels the result of [8] for finite joins. (iii) is a property of complete lattices.

PROPOSITION 3.2: (i) $f_{-}(q)$ is a complete join homomorphism.

(ii) $f_{-}(q)$ is complete with respect to $\Gamma$-meets for a cardinal $\Gamma$ if and only if each $q(x)$ is complete with respect to $\Gamma$-meets in $C(X)$ for each $x$.

(iii) $f_{-}(q)$ is bijective if and only if $q$ is indiscrete.

PROOF: (i) is from [8] while the proof of (ii) is similar to Theorem 4.2 of [8]. (iii) is dual to Proposition 3.1(iii).

From Proposition 3.2 one can observe that $f_{-}(q)$ is a complete lattice homomorphism if and only if $q$ is a pretopology. If $\Gamma$ and $\Omega$ are infinite cardinals with $\Gamma<\Omega$, by choosing the cardinal of $X$ large enough so that if $y \in X$ and $q(x)$ is discrete for $\mathrm{y} \neq \mathrm{x}$ and $\mathrm{q}(\mathrm{y})$ is closed with respect to $\Gamma$-meets but not $\Omega$-meets, then $\mathrm{f}_{-}(\mathrm{q})$ is a $\Gamma$-homomorphism that is not an $\Omega$-homomorphism.

Using the given four lattice operators, one can construct maps of certain sublattices of $\mathrm{C}(\mathrm{X})$ into the duals of their lattices of homomorphisms (with coordinatewise order). For example, if $L(X)$ is the lattice of limitierungs on $X$ and $P(X)$ the lattice of pretopologies, one can define $f_{T^{*}}^{*}: L(X) \rightarrow L^{L}$ by $f_{L}^{*}(q)(r)=$ $q * r$ and $f_{P}^{*}: P(X) \rightarrow P^{P}$ similarly, where $L^{L}\left(P^{P}\right)$ is the dual of the lattice of homomorphisms of $L(X)$ and $q * r$ is the relative pseudo-complement in $C(X)$. The succeeding two propositions follow directly from the definitions and properties of pseudo-complements and differences.

PROPOSITION 3.3: (i) $\mathrm{f}_{\mathrm{L}}{ }^{*}$ is a lattice embedding and a complete join homomorphism.

(ii) $\mathrm{f}_{\mathrm{P}}^{*}$ is a complete lattice embedding.

If $C^{C}(X, v)$ denotes the join semilattice of join-homomorphisms of $C(X)$ then 
the map $f_{-}: C(X) \rightarrow C^{C}(X, v)$ is $f_{-}(q)(r)=r-q$.

PROPOSITION 3.4: (i) $\mathrm{f}_{-}$is a complete meet homomorphism of $\mathrm{C}(\mathrm{X})$ into the dual of $C^{C}(X, v)$.

(ii) $f_{-}$is an embedding.

In a partially ordered set $(L, \leq)$, let $x<<y$ if and only if for every up-directed set $D, y \leq \sup D$ implies $x \leq d$ for some $d$ in $D$. Then from Scott [10], a complete lattice $L$ is continuous if $x=\sup \{y \in L \mid y<<x\}$ for all $x$ in $L$. The induced topology is that topology for which $U \subseteq L$ is open if $U$ is a terminal set and if $S \subseteq L$ is directed, sup $S$ exists and is in $U$, then $S \cap U \neq \phi$. Since a compactly generated lattice is continuous, we have from [8] that $C(X), L(X)$ and $P(X)$ are continuous with $C(X)$ and $L(X)$ having continuous duals. Since also from [10], a function between complete lattices is continuous in the induced topologies if and only if it is join-preserving, one has immediately from Propositions 3.1 and 3.2:

PROPOSITION 3.5: For any convergence structure $\mathrm{q}$ on $\mathrm{X}$ :

(i) $\mathrm{f}^{*}(\mathrm{q})$ is continuous if and only if each $\mathrm{q}(\mathrm{x}) \backslash\{\dot{\mathrm{x}}\}$ is prime with respect to joins in $F(X)$.

(ii) $f_{-}(q)$ is continuous.

PROPOSITION 3.6: $\mathrm{f}_{\mathrm{L}}^{*}$ and $\mathrm{f}_{\mathrm{P}}^{*}$ are continuous in the induced topologies.

Since join-prime elements $q$ determine when $f^{*}(q)$ is a homomorphism, one may note that if $q(x)$ is join-prime, there exists at most one ultrafilter $F$ not qconvergent to $x$. Also, the join-prime elements of $C(X)$ form a meet-sublattice of $C(X)$ but not a join-sublattice.

A number of special types of convergence structure lattices are continuous lattices by virtue of being retracts of $\mathrm{C}(\mathrm{X})$ in the induced topologies and Proposition 2.10 of $\left[10 \mid\right.$. Some examples are the lattices of $\mathrm{T}_{1}$-structures, pseudotopologies, locally bounded structures and locally compact structures which can 
be shown to be continuous by virtue of the standard modification maps.

In 181 , Theorem 5.1, the incorrect statement is made that the map $\phi$ of pretopological modification is a join homomorphism. If $q$ is the cofinite topology and $r$ is the finest convergence structure for which each principal ultrafilter converges to each point, then $\phi(q \vee r) \neq \phi q \vee \phi r$. Therefore $\phi$ cannot be used to show $P(X)$ is a continuous lattice.

\section{REFERENCES}

1. Carstens, A. M. The lattice of pretopologies on an arbitrary set S, Pacific J. Math. 29(1969) 67-71.

2. Feldman, W. A. Axioms of countability and the algebra $\mathrm{C}(\mathrm{X})$, Pacific $\underline{\mathrm{J}}$. Math. $47(1973) \quad 81-89$.

3. Fischer, H. R. Limesräume, Math. Ann. 137(1959) 269-303.

4. Hearsey, B. V. and D. C. Kent. Convergence structures, Portugaliae Math. $31(1972) \quad 105-118$.

5. Kent, D. C., G. D. Richardson and R. J. Gazik. T-regular closed convergence spaces, Proc. Amer. Math. Soc. 51(1975) 461-468.

6. Kent, D. C., K. McKennon, G. Richardson and M. Schroder. Continuous convergence in $\mathrm{C}(\mathrm{X})$, Pacific J. Math. 52(1974) 457-465.

7. Richardson, G. D. and D. C. Kent. The regularity series of a convergence space, Bull. Australian Math. Soc. 13(1975) 21-44.

8. Riecke, C. Complementation in the lattice of convergence structures, Pacific J. Math. 69(1977) 517-526.

9. Riecke, C. Ideals in convergence structure lattices, submitted.

10. Scott, D. Continuous lattices, Lecture Notes in Math., v. 274, SpringerVerlag, Berlin-New York, 1972.

11. Szasz, G. Introduction to lattice theory, Academic Press, New York, 1963. 


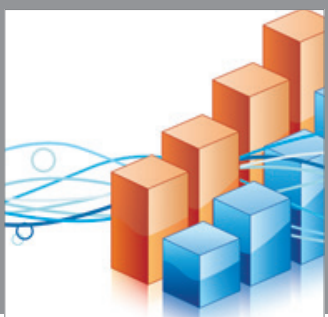

Advances in

Operations Research

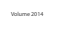

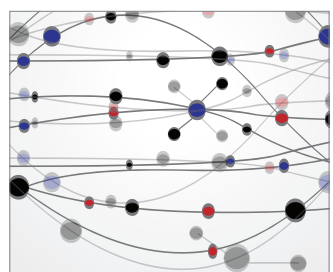

\section{The Scientific} World Journal
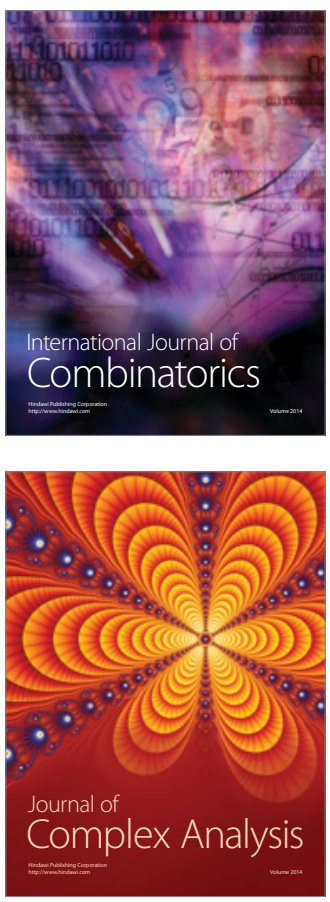

International Journal of

Mathematics and

Mathematical

Sciences
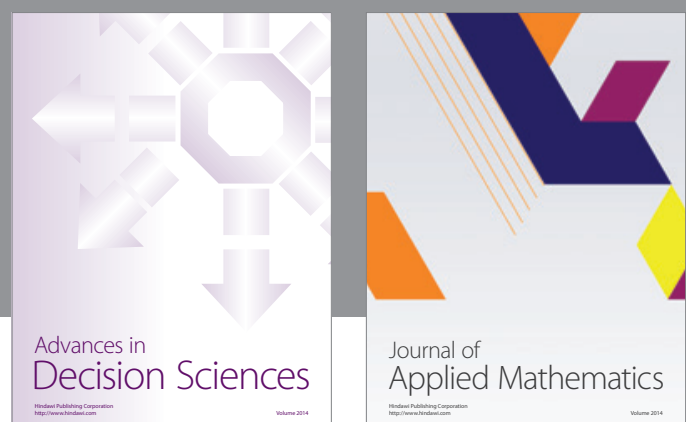

Journal of

Applied Mathematics
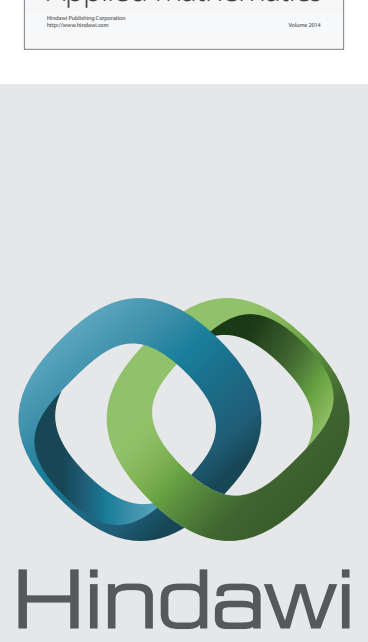

Submit your manuscripts at http://www.hindawi.com
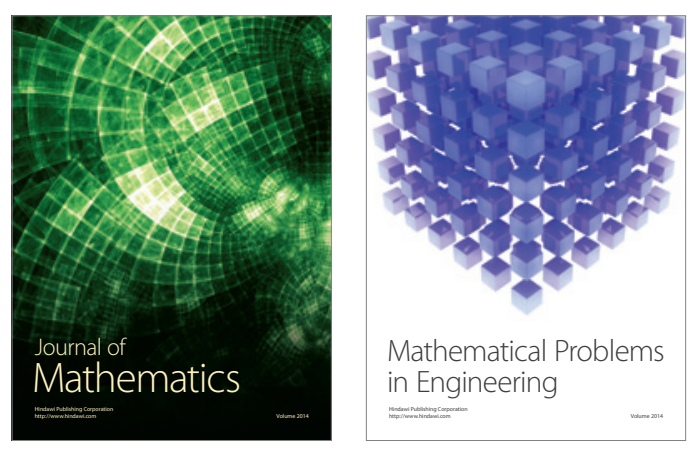

Mathematical Problems in Engineering
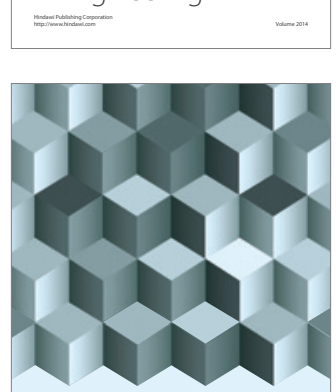

Journal of

Function Spaces
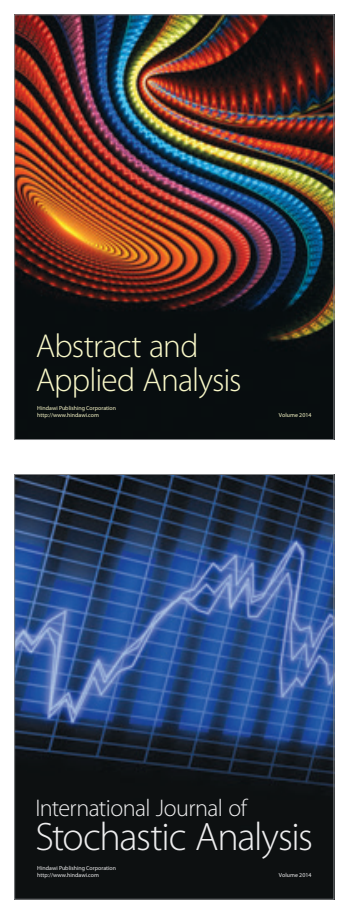

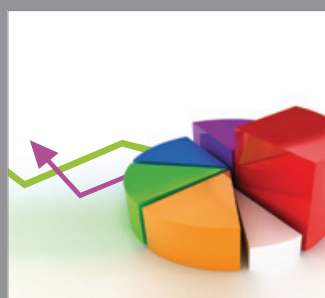

ournal of

Probability and Statistics

Promensencen
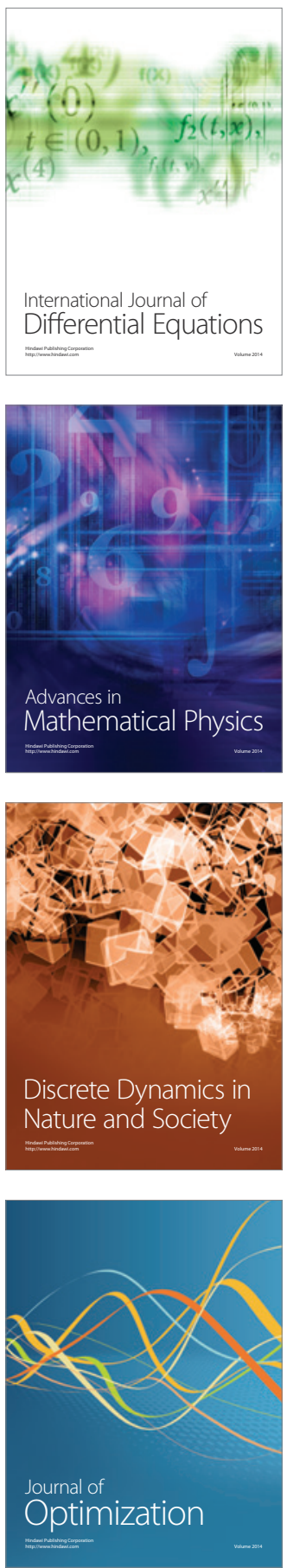